\title{
Automated Warning Reduces Error of Commission in Vigilance: A Study on Indian Adults
}

\author{
Dr. Amrita Panda*
}

\section{ABSTRACT:}

The spotlight regarding the concept of vigilance somehow was limited to the national security for decades. With technological advancement the concept of vigilance has attained importance in industrial set up. But vigilance as a cognitive aspect in regular life was somehow neglected throughout these years. An automated warning system is a sensory stimulation meant to reduce errors in highly loaded cognitive tasks. Previous research findings suggest that performance efficiency is uninfluenced by warning (Helton et. al., 2008) or a higher error of omission reported in the presence of warning signals (Helton et. al., 2011). But how it affects cognitive process like vigilance is rarely been explored. The present study attempts to reveal the effect of warning on vigilance. Participants were 95 adults chosen from metropolitan areas of Kolkata, India. Participant's Intelligence, processing speed and accuracy along with their psychiatric morbidity was controlled statistically. Finally, the participants were given a visual vigilance task using a software program (Panda \& Banerjee, 2011). Whenever the participants made two consecutive errors, an automated warning signal appeared. Results indicate a significant effect of automated warning on the false alarm scores. The means reflect a lower false alarm score when automated warning was given. The effect size indicates $35.3 \%$ of the change in false alarm score can be attributed by automated warning. The findings of the present study proposes if we can incorporate warning signals to provide feedback to the participants during performance of a visual vigilance task the performance accuracy can be increased. The finding of the study can be used in defense services, industrial set up as well as during performing day to day cognitive tasks that demand sustained attention or vigilance.

Keywords: Vigilance, Automated Warning, Error of Commission, Performance

*Project Fellow, Centre for the Study of Developmental Disability, Special Assistance Programme (DRS I), University Grants Commission, Department of Psychology, University of Calcutta, 92 Acharya Prafulla Chandra Road, Kolkata - 700009, West Bengal, India. )

*Editorial member of IJIP 


\section{INTRODUCTION}

Vigilance, or sustained attention, refers to the ability to monitor displays for stimulus events over prolonged periods of time. Sustained attention requires the ability to detect unpredictable and rare events over an extended period of time (Robbins, 1998), and includes vigilance, the state of alertness for infrequent and irregular events (Parasuraman et. al., 1998; Davies \& Parasuraman, 1982). The term 'vigilance' as applied to human behavior was coined by Sir Henry Head (1923), who referred to it as a state of maximum physiological and psychological readiness to react. However, the origin of modern vigilance research, as in many other areas of human factors, was in the Second World War.

Systematic research on vigilance began with Mackworth's (1948) pioneering research that suggested of a decline in performance efficiency over the period of watch, known as the vigilance decrement or the decrement function. It has been replicated in many studies and is the most commonly observed effect in vigilance or sustained attention research (Davies \& Parasuraman, 1982; Matthews et. al., 2000; Warm, 1993; Warm et. al., 2008). Vigilance tasks are useful for understanding the control of attention and the nature of attentional deficits (Broadbent, 1971; Manly et. al., 1999).

The traditional tasks used in studies of sustained attention are long detection tasks of scarcely occurring signals (Mackworth, 1948; Botella et al, 2001; Grier et. al., 2003). In many studies a vigilance decrement is found, indexed as a decline in the detection rate over time, showing its full strength after 20 to $35 \mathrm{~min}$. However, in other studies, using more complex tasks, no such decline of performance has been found (Warm, 1984). Several hypotheses have been described to account for the vigilance decrement. Some investigators (Stuss, 1995; Robertson et al, 1997) state that the vigilance decrement is a consequence of attentional withdrawal of the supervisory attentional system, due to underarousal caused by the insufficient workload inherent to typical vigilance tasks. Others (Temple et al, 2000; Grier et al, 2003) view the decrement as the result of a decrease of attentional capacity and thus as the impossibility to sustain the effort due to the mental workload.

Vigilance as a cognitive aspect has interested researchers for decades. But the spotlight regarding the concept of vigilance somehow was limited to the defense services and national security. With technological advancement the concept of vigilance has attained importance in industrial set up. But vigilance as a cognitive aspect in regular life is not explored too vividly and somehow was neglected till recent years. With the increased complexities of human life styles, exposure to numerous stimuli at any given point of time, it seems that successful accomplishment of cognitive tasks on regular basis demand individuals to be more vigilant than ever. Thus, the concept that was monopolized by the national securities or industrial settings to some extent is now viewed as a global necessity of every single individual to ensure success in all aspects of daily life. Starting from crossing a busy road to attend a complex classroom lecture, to understand an individual's exact personality pattern amongst his/her apparent behavioral complexities, to be successful in a debate competition, individuals not only require sustained 
attention but they are required to be extremely vigilant to successfully accomplish the cognitive processes. The present study thus aims to explore vigilance as a cognitive aspect of day to day life and highlight if automated warning affect vigilance performance or not.

The importance of vigilance has vaulted to the forefront of current social concerns regarding detection of terrorist activities (Hancock \& Hart, 2002). Such tasks characterize many humanmachine interactions in automated systems (Howell, 1993; Nickerson, 1992). Sustained attention also plays a critical role in many applied settings, such as process and quality control, medical monitoring, and baggage inspection (Hancock \& Hart, 2002; Wickens \& Hollands, 2000).Yet until recently, psychologists and human factors researchers typically viewed vigilance tasks by virtue of their repetitiveness and simplicity as tedious and cognitively undemanding (Heilman, 1995). However, studies using the NASA-Task Load Index (NASA-TLX; Hart \& Staveland, 1988) have shown that the mental workload of vigilance tasks is substantial (Deaton \& Parasuraman, 1993; Warm et. al., 1996).

An automated warning system is a sensory stimulation meant to reduce errors in highly loaded cognitive tasks. An automated warning system is supposed to give an alert when an individual is about to meet a danger. There is significant interest among traffic management personnel in the use of automated warning systems to provide drivers with real-time information on hazardous conditions related to traffic, limited visibility, or roadway obstructions. However, the effectiveness of such systems in safety improvements has not yet been well quantified. With the increase in automation, the concept of automated warning had been increasingly used in industrial setup. But how it affects vigilance as a cognitive process is not yet explored extensively. The present study attempts to throw light on the interrelationship of vigilance and automated warning and aims to reveal if warning actually reduces performance decrement, or decreases performance accuracy inducing anxiety or reduces performance decrement initially, but heightens anxiety that result in performance decrement in long run. So that it can be used more widely during vigilance tasks or suggestions can be made regarding more cautious use of automated warning during vigilance task.

Previous research findings suggest that performance efficiency is uninfluenced by warning (Helton et. al., 2008) or introduction of warning signals reduced RT (Ponsford \& KInsella, 1992; Van Zomeren et. al., 1984). Previous research shows that with the introduction of knowledge of result (KR) performance in vigilance task was more stable over time in the KR condition, performance declined in the no-KR condition (Shaw et. al. 2009). Helton et. al. (2011) claims that with the use of warning response times were faster, errors of commission lower, but errors of omission higher in the reliable-warning task in comparison with the no-warning. In another intriguing study of knowledge of results, Loeb and Schmidt (1960) found suggestive evidence that false knowledge of results also improves subject's performance on auditory vigilance tasks. Again, Szalma et. al. (2006) investigated the impact of knowledge of results (KR) format on the performance and stress associated with a vigilance task and revealed that there is a trade-off in the effectiveness of KR in reducing false alarms and misses. 
On the backdrop of these research findings that present study aims to explore the effect of automated warning on vigilance in clinically normal participants.

\section{METHOD}

\section{Participants}

The participants were selected using random sampling technique. Participants were 95 clinically normal individuals (Mean age $=22.62, \mathrm{SD}=6.51$ ) chosen from higher secondary schools or colleges or from professional institutions of metropolitan areas of Kolkata, India. Participants were instructed adequately and were briefed about the nature of the study. Written permission was taken from the institutions they belonged to, at the same time participant's individual consent was also taken. The samples were assessed according to their socio-economic status, intelligence capacity, general health and clerical speed and accuracy scores. Measures on all these variables were controlled statistically.

\section{Selection Criteria}

$\underline{\text { Inclusion Criteria }}$

1. Age between $18-35$ years

2. Belong to middle or upper-middle socioeconomic status

3. Motivation (as per verbal report) and available time to participate in experimental sessions.

\section{$\underline{\text { Exclusion Criteria }}$}

1. Presence of any past psychiatric illness or organic disorder or chronic illness.

2. Presence of any mental disability.

3. Presence of any physical disability.

4. Lack of motivation for the treatment or lack of time availability to attend the sessions.

\section{Tools Used}

Information Schedule: An information schedule is used to collect personal and familial information about the subject. The schedule was prepared by the present investigator as per the requirements of the present study.

GHQ: A Bengali adaptation (Basu and Dasgupta, 1996) of the 30-item General Health Questionnaire (GHQ) of Goldberg (1972) was used as a unidimensional measure for 
screening functional psychiatric illness to detect non-psychotic psychiatric disturbances in a variety of settings

Standard Progressive Matrices: Standard Progressive Matrices by J. C. Raven (1938) was used to measure the intellectual functioning of the participants.

DAT-Speed and Accuracy Test: The Clerical Speed and Accuracy subtest of Differential Aptitude Test (DAT) developed by G. K. Bennett, H. G. Seashore and A. G. Wesman (1947) was used to measure individual's speed and accuracy in cognitive tasks in normal participants.

A software program: The participants were given a visual vigilance task using a software program (Panda et. al., 2011). They were asked to detect a target stimulus, that is, a small pink rounded rectangle or small yellow rounded rectangle in respective trials among randomly occurring buffer stimuli of different color, size and shape. The target stimulus was discriminable from the non-target stimuli. Participants were asked to press a particular key on computer whenever they see a target stimulus to occur on computer screen. Whenever participants made two consecutive errors in target stimulus detection, a warning signal occurred. The warning was visual in nature and appeared as a big red colored star in the middle of the screen. The response of the participants were measured in terms of successful detection of target stimuli (hit), failure in detection of a target stimuli (miss) and response given to a buffer stimuli in lieu of target stimuli (false alarm).

\section{Procedure}

- First, the samples, following the selection criteria was randomly chosen for the study. All the participants were explained about the present study and were assured about confidentiality of their responses and identity.

- The participants were given the information schedule, GHQ, SPM, DAT Speed and Accuracy. DAT Speed and Accuracy was administered as the stimulus was a software program and response involved computer application. It was assumed that processing speed accuracy would be a variable to control to observe only the effect of warning.

- Then the vigilance task was given to each of the samples using the software program.

- For the vigilance task there was a Control Condition (CC) and an Experimental Conditions $\left(\mathrm{EC}_{1}\right)$. In the Control Condition only the visual vigilance task was given to the samples.

- In $\mathrm{EC}_{1}$ along with the vigilance task, whenever the subject made two consecutive errors an automated warning was given to warn them about their response errors in the assigned task and to suggest that they need to be more cautious about their subsequent response choices.

- The response of the participants from both the conditions was taken into account in terms of hit scores and false alarm scores.

Data Computation and Statistical Analyses 
At first, the data were tabulated following the scoring key of each checklist and for each individual separately. The entire data was then analyzed using SPSS 21.

\section{RESULT}

Table: 1 showing the descriptive statistics of the distribution of DV measures of Normal group

\begin{tabular}{|l|r|r|r|r|}
\hline \multicolumn{1}{|l|}{} & C_HIT & C_FA & E1_HIT & E1_FA \\
\hline N & 95 & 95 & 95 & 95 \\
\hline Mean & $\mathbf{1 1 9 . 4 9}$ & $\mathbf{3 9 . 9 1}$ & $\mathbf{1 1 6 . 8 3}$ & $\mathbf{3 0 . 6 0}$ \\
\hline Std. Error of Mean & 2.577 & 2.125 & 2.666 & 1.711 \\
\hline Median & $\mathbf{1 2 6 . 0 0}$ & $\mathbf{3 3 . 0 0}$ & $\mathbf{1 2 0 . 0 0}$ & $\mathbf{2 6 . 0 0}$ \\
\hline Mode & 101 & 26 & 134 & 69 \\
\hline Std. Deviation & 25.114 & 20.713 & 25.982 & 16.673 \\
\hline Skewness & -.950 & .931 & -.577 & .793 \\
\hline Kurtosis & .392 & .092 & -.606 & .078 \\
\hline Minimum & 51 & 7 & 59 & 4 \\
\hline Maximum & 155 & 90 & 154 & 69 \\
\hline
\end{tabular}

As per the proximity of the mean and median value of the DV measures and the observed skewness and kurtosis of the DV measurement the data is considered for parametric analysis.

\section{Role of Automated Warning on Vigilance in Normal Participants}

Does automated warning improves performance in vigilance task? This section explores the relationship of automated warning with vigilance. In the control condition (CC) no warning was given, but a warning is used in the experimental condition $\left(\mathrm{EC}_{1}\right)$. The performance in vigilance task has been measured through correct detection of target stimuli (hit score) and scores on detection of target stimuli when it is in fact a non-target stimuli (false alarm score). The relationships of automated warning with other covariates are also assessed. Age, socio economic status (SES) of individuals along with their general health (GH), intellectual capacity (IQ), and speed and accuracy (SA) was measured. Initially it was planned that these variables will be taken as controls in the study but during actual data collection it came up that variations in these variables are supposed to turn up with new relationships in the domain of vigilance. The mean and standard deviations of the above mentioned variables are depicted in Table 2. 
Table: 2 Descriptive Statistics of Control variables

\begin{tabular}{|l|r|r|}
\hline & Mean & SD \\
\hline Age & 22.62 & 6.512 \\
\hline SES & 2.77 & 1.026 \\
\hline GHQ & 7.75 & 6.046 \\
\hline IQ & 39.79 & 11.200 \\
\hline WM & 6.74 & 1.151 \\
\hline SA & 53.40 & 14.261 \\
\hline C_HIT & 119.49 & 25.114 \\
\hline C_FA & 39.91 & 20.713 \\
\hline
\end{tabular}

The first step in exploring the effect of automated warning was to look at the relationships between variables (Table: 3 ). IQ and speed and accuracy were found to have significant positive correlation with the hit scores and a significant negative correlation with the false alarm scores. No other variable that was assumed to effect vigilance initially had any relationship with vigilance. Intellectual functioning is found to be significantly correlated with speed and accuracy. Socioeconomic status has been found to be positively correlated with intellectual capacity and working memory functioning and negatively with general health. Age is found to be negatively correlated with speed and accuracy.

Table: 3 Correlation Studies of Control variables with vigilance

\begin{tabular}{|l|c|c|c|c|c|c|c|}
\hline & Age & SES & GH & IQ & SA & C_HIT & C_FA \\
\hline Age & 1 & & & & & & \\
\hline SES & .106 & 1 & & & & & \\
\hline GH & -.059 & $\mathbf{- . 2 0 9}^{*}$ & 1 & & & & \\
\hline IQ & .003 & $\mathbf{. 3 0 1}^{* *}$ & -.056 & 1 & & & \\
\hline SA & $\mathbf{. . 3 6 0}^{* *}$ & .161 & -.008 & $\mathbf{. 4 4 9}^{* *}$ & 1 & & \\
\hline C_HIT & -.014 & .160 & -.120 & $\mathbf{. 2 9 3}^{* *}$ & $\mathbf{. 3 0 9}^{* *}$ & 1 & \\
\hline C_FA & .012 & $-.280^{*}$ & .124 & $\mathbf{. . 2 6 9}^{* *}$ & $\mathbf{- . 3 0 1}^{* *}$ & $\mathbf{. . 2 0 2}^{*}$ & 1 \\
\hline
\end{tabular}

\section{Role of Warning on Hit score of Vigilance in Normal Participants}

To find out the effect of automated warning on hit scores of vigilance a repeated measure ANCOVA was done. As speed and accuracy and IQ both correlate with vigilance scores they are considered to be covariates. Though a correlation exist between these covariates still as the value 
of correlation is less than .5 it's considered as low correlation and hence they fulfill the criteria of being covariates in a repeated measure ANCOVA design.

Table: 4 Showing the Mean, SD \& F value of Control and Experimental Condition

\begin{tabular}{|c|c|c|c|c|c|c|c|}
\hline Conditions & Mean & SD & $\mathbf{N}$ & $\begin{array}{l}\text { Wilk's } \\
\text { Lamda } \\
\text { Value }\end{array}$ & $\mathbf{F}$ & df & Sig. \\
\hline No Warning & 119.49 & 25.114 & 95 & .971 & 2.840 & 1 & .095 \\
\hline Warning & 116.83 & 25.982 & 95 & & & & \\
\hline
\end{tabular}

Table 4 shows no significant effect of automated warning was found on hit scores of vigilance in normal participants while controlling for the covariates. The Mauschley's W value equals to 1, indicating that the assumption of sphericity is met in repeated measure design.

\section{Role of Warning on False alarm (FA) score of Vigilance in Normal Participants}

To find out the effect of automated warning on false alarm scores of vigilance a repeated measure ANCOVA was done. As speed and accuracy both correlate with the false alarm scores of vigilance they are again considered to be covariates.

Table 5 shows significant effect of automated warning on false alarm scores of vigilance in normal participants while controlling for the covariates. Here, unlike hit scores, the mean scores indicate significant increase in performance efficiency with the introduction of warning in vigilance task. The Mauchly's W value (1) indicates that the assumption of sphericity has been met in repeated measure design.

Table: 5 Showing Mean, SD \& F value of Control and Experimental Condition

\begin{tabular}{|l|c|c|c|c|c|c|c|c|}
\hline Conditions & Mean & SD & N & $\begin{array}{c}\text { Wilk's } \\
\text { Lamda } \\
\text { Value }\end{array}$ & F & df & Sig. & \\
\hline No Warning & 39.91 & 20.713 & 95 & .647 & 51.336 & 1 & .000 & .353 \\
\hline Warning & 30.60 & 16.673 & 95 & & & & & \\
\hline
\end{tabular}

The effect size of .353 reflects that $35.3 \%$ variability in the false alarm score can be attributed to introduction of warning signal in experimental condition.

To further explore the effects of the covariates preliminary analysis was done (Table 6) to have a more clear idea about the nature of the covariates. To find out the effect of different levels of IQ and speed and accuracy both of the variables were blocked according to their first and third 
quartile. Hence the blocking resulted in two IQ groups - high and low and two speed and accuracy groups - high and low.

Table: 6 Descriptive Statistics of Covariates

\begin{tabular}{|l|r|r|r|}
\hline & & IQ & Speed Accuracy \\
\hline N & 95 & 95 \\
\hline Mean & 39.87 & 53.40 \\
\hline Mode & 41.00 & 51.00 \\
\hline Std. Deviation & 40 & 47 \\
\hline Skewness & 10.979 & 14.261 \\
\hline Kurtosis & -.694 & .359 \\
\hline Minimum & .033 & -.395 \\
\hline Maximum & 13 & 20 \\
\hline Percentiles & 25 & 59 & 89 \\
\cline { 2 - 5 } & 50 & $\mathbf{3 2 . 0 0}$ & $\mathbf{4 3 . 0 0}$ \\
\cline { 2 - 5 } & 75 & 41.00 & 51.00 \\
\hline
\end{tabular}

To have a look at the effect of the covariates it was found that the effect of intellectual functioning was not significant, whereas the effect of speed and accuracy is significant (Table 7), reflecting that the different levels of speed accuracy act differently in no-warning to warning conditions in vigilance task. The $\eta_{\mathrm{p}}^{2}$ indicate .083 for speed and accuracy.

Table: 7 Showing Inferential Statistics of effect of covariates on false alarm scores of vigilance in normal participants

\begin{tabular}{|l|c|c|c|c|c|}
\hline Source & df & Mean Square & F & Sig. & $\begin{array}{c}\text { Partial Eta } \\
\text { Squared }\end{array}$ \\
\hline IQ & 1 & 811.315 & 1.490 & .225 & .016 \\
\hline SA & 1 & 4511.753 & 8.288 & .005 & .083 \\
\hline
\end{tabular}

Next, with the two speed accuracy groups two repeated measure analysis was run consecutively. The effect of the warning conditions was statistically significant in both low $(\mathrm{F}=9.363, \mathrm{p}=0.006)$ and high $(\mathrm{F}=14.284, \mathrm{p}=.001)$ speed and accuracy groups. For both the groups the false alarm score decreased with the introduction of warning (Table 8). But for the high speed accuracy group the mean score was much lower than that of low speed accuracy group. 
Table: 8 Showing Descriptive and Inferential Statistics of effect of automated warning on false alarm scores of vigilance between both the Speed Accuracy groups in normal participants

\begin{tabular}{|c|c|c|c|c|c|c|c|c|c|}
\hline $\begin{array}{l}\text { SA } \\
\text { GROUP }\end{array}$ & Conditions & Mean & SD & $\mathbf{N}$ & $\begin{array}{c}\text { Wilk's } \\
\text { Lamda } \\
\text { Value }\end{array}$ & $\mathbf{F}$ & df & Sig. & $\begin{array}{l}\text { Partial } \\
\text { Eta } \\
\text { Squared }\end{array}$ \\
\hline \multirow[t]{3}{*}{ Low } & No Warning & 48.58 & 24.624 & 24 & .711 & 9.363 & 1 & .006 & .289 \\
\hline & Warning & 38.92 & 18.418 & 24 & & & & & \\
\hline & Total & 43.75 & 21.521 & 24 & & & & & \\
\hline \multirow[t]{3}{*}{ High } & No Warning & 29.62 & 13.147 & 24 & .617 & 14.284 & 1 & .001 & .383 \\
\hline & Warning & 21.12 & 8.502 & 24 & & & & & \\
\hline & Total & 25.37 & 10.8245 & 24 & & & & & \\
\hline
\end{tabular}

The effect size $\left(\eta_{\mathrm{p}}{ }^{2}=0.289\right)$ measure indicates $28.9 \%$ of the change in false alarm score can be attributed by automated warning in low processing speed group, whereas for high speed accuracy group it increases to $38.3 \%$, i.e., $38.3 \%$ of the variability in false alarm scores can be explained by warning in high processing speed group.

\section{DISCUSSION}

The findings of the present study indicate increment in performance accuracy of vigilance task in presence of automated warning. Though the error of omission scores remain unaffected by automated warning, it was evident that introduction of waning signals reduced the error of commission scores. The effect size measure reflects quite a large effect of automated warning on the error of commission scores according to Cohen's (1988) guideline. Further, intellectual capacity of an individual at the same time processing speed and accuracy of a clinically normal participant both are found to be correlated with vigilance.

The present study refutes the suggestion of the previous finding that vigilance performance remain unaffected with the use of warning mechanism (Helton et. al., 2008). Instead of inducing anxiety to have a deteriorating effect on performance, knowledge of result actually helps clinically normal individuals to perform more accurately in vigilance task. It is evident from the present study though introduction of warning could not improve hit scores in vigilance it restores a cognitive alertness in the individual so that error of commission scores decreased. Even if the warning signal induces stress, it works as a eustress to the individual and improves vigilance performance. The optimum environment for vigilance tasks is rather more arousing than the optimum for tasks which are intrinsically more interesting (Poulton, 1977). The findings of the 
present study support the suggestions of previous findings (Shaw et. al. 2009; Helton et. al., 2011) that warning improves vigilance.

Vigilance had traditionally been associated with low cognitive demand and vigilance decrement with a decline in arousal pursuant to the low cognitive demand (Frankmann \& Adams, 1962) but these views are no longer widely held. More recent studies indicate that vigilance is hard work, requiring the allocation of significant cognitive resources, and inducing significant levels of stress (Parasuraman \& Davies, 1977). Reductions in arousal generally correspond to reductions in vigilance. Arousal is a component of vigilance, though not, as once believed, the sole source of the main effect of the vigilance decrement (Moruzzi \& Magoun, 1949) As such, subcortical brain regions associated with arousal play a critical role in the performance of vigilance tasks. Because the amygdala plays an important role in the recognition of emotional stimuli, it appears to be an important brain structure in the regulation of vigilance (Sternberg, 2009).

Further, the present study reveals a probable relationship between vigilance and both intellectual capacity and processing speed accuracy, suggesting that increase in intellectual capacity and processing speed improves vigilance. It could be noticed that individuals with high processing speed got more benefited by warning as compared to their counterparts. Research indicate that measures of intelligence are significantly correlated with mental speed and that for some measures this relationship shows a trend toward strengthening as the complexity of the speeded tasks increase (Leah \& Vernon, 2008). Further, individuals with faster perceptual speed tend to also have faster processing in general on mundane tasks that require them to identify a basic stimulus and respond. Vernon argued that processing information faster neurologically may permit for one to learn more. However, although research is hinting towards a connection between neurological processing speed and intelligence, there are no definitive answers as to why of yet. Many leading researchers believe that it is a result of overall more efficient cognitive processing. Hence, the participants who were high in processing speed are more efficient to receive the warning signals and make the most use of it. Thus the study emphasizes the role of processing speed and accuracy in successful accomplishment of vigilance task.

Overall the study indicates if we can incorporate warning signals to provide feedback to the participants during performance of a visual vigilance task the performance accuracy can be increased. The finding of the study can be used in defense services, industrial set up as well as during performing day to day cognitive tasks that demand sustained attention or vigilance.

\section{KEY POINTS}

- Vigilance as a cognitive aspect in day to day life has not been explored too vividly in the history of vigilance research.

- The present study aims to explore the effect of automated warning on vigilance. 
- Results reflect no significant effect of warning on error of omission scores but error of commission scores were significantly reduced with the introduction of warning in vigilance task.

- The study suggests if auto-suggestions or warning systems can be incorporated in regular cognitive tasks that require vigilance, performance accuracy of normal adults can be improved.

\section{REFERENCE}

1. Basu, S. \& Dasgupta, S. K. (1996). Adaptation of GHQ-28 into Bengali. Department of Applied Psychology. Kolkata: University of Calcutta.

2. Bennett, G. K., Seashore, H. G., \& Wesman, A. G. (1947). Differential Aptitude Tests, Form A. New York: Psychology Corporation.

3. Botella J., Contreras M.J., Shih P.C., \& Rubio V. (2001). Two short tests fail to detect vigilance decrements. European Journal of Psychological Assessment, 17, 48-55.

4. Broadbent, D. E. (1971). Decision and stress. New York: Academic Press.

5. Cohen, J. (1988). Statistical Power Analysis for the Behavioral Sciences (2nd ed.), New Jersey: Lawrence Erlbaum Associates.

6. Davies, D. R., \& Parasuraman, R. (1982). The Psychology of Vigilance. London: Academic.

7. Deaton, J. E., \& Parasuraman, R. (1993). Sensory and cognitive vigilance: Effects of age on performance and mental workload. Human Performance, 6, 71-97.

8. Frankmann, J.P. \& Adams, J.A. (1962). Theories of Vigilance. Psychological Bulletin, $59,257-272$.

9. Goldberg, D. P. (1972). The detection of psychiatric illness by questionnaire. London, Oxford University Press.

10. Grier, R. A., Warm, J. S., Dember, W. N., Matthews, G., Galinsky, T. L., Szalma, J. L. et al. (2003). The vigilance decrement reflects limitations in effortful attention, not mindlessness. Human Factors, 45, 349-359

11. Hancock, P. A., \& Hart, S. G. (2002). Defeating terrorism: What can human factors/ergonomics offer? Ergonomics in Design, 10(1), 6-16.

12. Hart, S., \& Staveland, L. (1988). Development of the NASA-TLX (Task Load Index): Results of empirical and theoretical research. In P. A. Hancock \& N. Meshkati (Eds.), Human mental workload (pp. 139-183). Amsterdsam: North-Holland.

13. Head, H. (1923). The conception of nervous and mental energy: II. Vigilance: A physiological state of the nervous system. British Journal of Psychology, 14, 126-147.

14. Helton, S. W.; Shaw, T.; Warm, J. S.; Matthews, G. \& Hancock, P. (2008). Effects of warned and unwarned demand transitions on vigilance performance and stress. Anxiety, Stress, \& Coping, 21(2): 173 - 184.

15. Helton, W.S., Head, J. \& Russell, P.N. (2011). Reliable- and unreliable-warning cues in the sustained attention to response task. Experimental Brain Research, 209, pp. 401-407. 
16. Heilman, K. M. (1995). Attentional asymmetries. In R. J. Davidson \& K. Hugdahl (Eds.), Brain asymmetry (pp. 217-234). Cambridge: MIT Press.

17. Howell, W. C. (1993). Engineering psychology in a changing world. Annual Review of Psychology, 44, 231-263.

18. Loeb, M., \& Schmidt, E. A. (1960). Influence of time on task and false information on efficiency of responding to pure tones. AMRL 42 6.. Aerospace Medical Research Laboratories, Wright-Patterson AFB, Ohio.

19. Mackworth, N. H. (1948). The breakdown of vigilance during prolonged visual search. Quarterly Journal of Experimental Psychology, 1, 6-21.

20. Manly, T., Robertson, I. H., Galloway, M., \& Hawkins, K. (1999). The absent mind: Further investigations of sustained attention to response. Neuropsychologia, 37, 661-670.

21. Matthews, G., Davies, D. R., Westerman, S. J., \& Stammers, R. B. (2000). Human performance: Cognition, stress and individual differences. East Sussex, UK:Psychology Press.

22. Moruzzi, G. and Magoun, H.W. (1949) Brain stem reticular formation and activation of the EEG. Clinical Neurophysiology I: 455-473

23. Nickerson, R. S. (1992). Looking ahead: Human factors challenge in a changing world. Mahwah, NJ: Erlbaum.

24. Panda, A.; Bhattacharya, C. \& Banerjee, M. (2011). Does absence of hearing ability enhance visual vigilance? It depends on task complexity. Psychological Studies, 56, 3, 266-270.

25. Parasuraman, R. \& Davies, D.R. (1977). A taxonomic analysis of vigilance. In R.R. Mackie, (ed.) Vigilance: Theory, operational performance and physiological correlates, (pp 559-574) New York: Plenum.

26. Parasuraman, R., Warm, J. S., \& See, J. E. (1998). Brain systems of vigilance. In R. Parasuraman (Ed.), The attentive brain (pp. 221-256). Cambridge, MA: MIT Press.

27. Ponsford, J. \& Kinsella, G. (1992). Attentional deficits following closed head injury. Journal of Clinical Experimental Neuropsychology, 14:822-838.

28. Poulton, E. C. (1977). Arousing Stresses Increase Vigilance. NATO Conference Series, Volume 3, pp 423-459

29. Raven, J. C. (1938). Progressive matrices: A perceptual test of intelligence. London: H.K. Lewis \& Co. Ltd.

30. Robbins T. W. (1998) Arousal and attention: psychopharmacological and neuropsychological studies in experimental animals. In: The Attentive Brain (ed. R. Parasuraman), pp. 189-220. The MIT Press, Cambridge, MA.

31. Shaw, T. H., Warm, J. S., Finomore, V. S., Tripp, L., Matthews, G., Weiler, E., \& Parasuraman, R. (2009). Effects of sensory modality on cerebral blood flow velocity during vigilance. Neuroscience Letters, 461, 207-211. 
32. Sheppard, L. D. \& Vernon, P. A. (2008). Intelligence and speed of informationprocessing: A review of 50 years of research. Personality and Individual Differences. Volume 44, Issue 3, Pages 535-551.

33. Sternberg, R. (2009). Cognitive Psychology. Belmont, CA: Wadsworth, Cengage Learning. p. 145.

34. Stuss, D. T. (1995). A Sensible Approach to Mild Traumatic Brain Injury. Neurology, 45, 1251-1252.

35. Szalma, J. L., Hancock, P. A., Warm, J. S., Dember, W. N., \& Parsons, K. S. (2006). Training for vigilance: Using predictive power to evaluate feedback effectiveness. Human Factors, 48, 682-692.

36. Temple, J. G., Warm, J. S., Dember, W. N., Jones, K. T. S., LaGrange, C. M., \& Matthews, G. (2000). The effects of signal salience and caffeine on performance, workload, and stress in an abbreviated vigilance task. Human Factors, 42, 183-194.

37. Van Zomeren, A. H., Brouwer, W. H., \& Deelman, B. G. (1984) Attentional deficits: the riddles of selectivity, speed, and alertness. In: Brooks N (ed) Closed head injury: psychological, social, and family consequences. Oxford University Press, New York, pp 74-107

38. Warm, J. S. (1993). Vigilance and target detection. In B. M. Huey \& C. D. Wickens (Eds.), Workload transition: Implications for individual and team performance (pp. 139 170). Washington, DC: National Academy Press.

39. Warm, J. S., Matthews, G., \& Finomore, V. S. (2008). Workload, stress, and vigilance. In P. A. Hancock \& J. L. Szalma (Eds.), Performance under stress (pp. 115-141). Brookfield, VT: Ashgate.

40. Warm, J. S., Parasuraman, R., \& Matthews, G. (2008). Vigilance Requires Hard Mental Work and Is Stressful. Human Factors, Vol. 50, No. 3, June 2008, pp. 433-44.

41. Warm, J. S., Dember, W. N., \& Hancock, P. A. (1996). Vigilance and workload in automated systems. In R. Parasuraman \& M. Mouloua (Eds.), Automation and human performance: Theory and applications (pp. 183-200) Mahwah, NJ: Erlbaum.

42. Wickens, C. D., \& Hollands, J. G. (2000). Engineering psychology and human performance $\left(3^{\text {rd }} \quad\right.$ ed.). Upper Saddle River, NJ: Prentice Hall. 